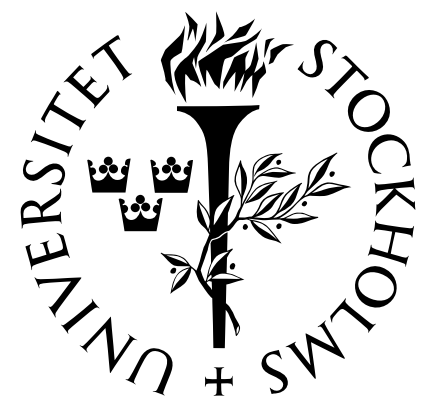

\title{
On Equi-transmitting Matrices
}

Pavel Kurasov and Rao Ogik

Research Reports in Mathematics

Number 1, 2014

Department of Mathematics

STOCKHOLM UNIVERSITY 
Electronic versions of this document are available at http://www.math.su.se/reports/2014/1

Date of publication: February 24, 2014

2010 Mathematics Subject Classification: Primary 34L25, 81U40, Secondary 35P25, $81 \mathrm{~V} 99$

Keywords: Quantum graphs, vertex scattering matrix, equi-transmitting matrices

Postal address:

Department of Mathematics

Stockholm University

S-106 91 Stockholm

Sweden

Electronic addresses:

http://www.math.su.se/

info@math.su.se 


\title{
ON EQUI-TRANSMITTING MATRICES
}

\author{
PAVEL KURASOV AND RAO OGIK
}

\begin{abstract}
Equi-transmitting scattering matrices are studied. A complete description of such matrices up to order six is given. It is shown that the standard matching conditions matrix is essentially the only equi-transmitting matrix for orders 3 and 5. For orders 4 and 6 , there exists other equi-transmitting but all such matrices have zero trace.
\end{abstract}

\section{Introduction}

From quantum physics it is known that, for objects with finite number of scattering channels, the scattering of waves at a node is described by unitary matrices. The unitarity of the scattering matrix means that the system is conservative.

The transition probabilities between different channels are given by modulus squares of the corresponding matrix elements $\left|s_{i j}\right|^{2}$. Therefore a vertex scattering matrix $S_{V}$ determines a system where all channels are equivalent if and only if $\left|s_{i j}\right|^{2}=\left|s_{l m}\right|^{2}, i \neq j, l \neq m$. This implies that even diagonal elements have the same value $\left|s_{i i}\right|^{2}=\left|s_{j j}\right|^{2}, i \neq j$. Such matrices are called equi-transmitting matrices.

This classical problem has found renaissance in quantum graphs where the transition through vertices is described by vertex scattering matrix $S_{V}(k)$. The matrix has the following explicit energy dependence

$$
S_{V}(k)=\frac{(k+1) S_{V}(1)+(k-1) I}{(k-1) S_{V}(1)+(k+1) I} .
$$

Here $S_{V}(1)$ is the vertex scattering matrix for $k=1$ and can be considered as a parameter describing all possible vertex conditions $[15,14]$. We are interested in equi-transmitting matrices $S_{V}(k)$ and so $S_{V}(1)$ has to be equi-transmitting. If $S_{V}(1)$ is not only unitary, but also Hermitian, then $S_{V}(k)$ does not depend on the energy $k^{2}$. Therefore for $S_{V}(k)$ to be equi-transmitting it is sufficient that $S_{V}(1)$ is not only equi-transmitting and unitary but also Hermitian.

A widely used vertex condition is the so called standard (Kirchoff) matching conditions (SMC) on the functions at a vertex $V$ are

$$
\left\{\begin{array}{l}
u \text { is continuous at every vertex } V \\
\sum_{x_{j} \in V_{m}} \partial_{n} u\left(x_{j}\right)=0
\end{array}\right.
$$

The corresponding vertex scattering matrix is

$$
\left(\begin{array}{cccc}
\frac{2}{v}-1 & \frac{2}{v} & \frac{2}{v} & \cdots \\
\frac{2}{v} & \frac{2}{v}-1 & \frac{2}{v} & \cdots \\
\frac{2}{v} & \frac{2}{v} & \frac{2}{v}-1 & \\
\vdots & \vdots & & \ddots
\end{array}\right),
$$

where $v$ is the degree of the vertex. We see that it is equi-transmitting. 
Turek and Cheon have studied equi-transmitting matrices in [19]. Their construction is by means of the Hadamard and conference matrices and they have given several examples of equi-transmitting matrices.

The aim of this study is to give a complete description of all equi-transmitting matrices up to dimension 6. In section two we describe the general structure of an equi-transmitting matrix and the various transformations that preserve equi-transmission properties. In section three we describe the possible values of $r$ and $t$. It is shown that $r, t \in[0,1]$ with the interesting cases being $r, t \in(0,1)$. In section four we consider several examples of equitransmitting matrices including the one corresponding to SMC. Sections five and six deal with the construction of such matrices in dimensions 2 to 6 for $r, t \in(0,1)$. The following results are obtained:

- When $n=3$ and $n=5$, the only equi-transmitting matrix are those corresponding to the SMC-matrix.

- When $n=2,4,6$ and $\operatorname{Tr}(S)=0$, other equi-transmitting matrices exist besides those generated by the SMC-matrix.

Definition 1. An $n \times n$ matrix $S$ is equi-transmitting (ET-matrix) if the following hold

1. $S=S^{*}=S^{-1}$,

2. $\left|s_{i i}\right|=\left|s_{j j}\right|, \quad i \neq j$,

3. $\left|s_{i j}\right|=\left|s_{l m}\right|, \quad i \neq j, l \neq m$.

\section{Formulation and transformations}

One of the important notion in quantum graphs is the vertex scattering matrix. For a vertex $V$ with valency $n$, the associated vertex scattering matrix $S_{V}(k)$ is an $n \times n$ unitary matrix that describes how waves are scattered at that vertex. If the matrix $S_{V}(1)$ is plugged into the matching conditions equation $i(S-I) \vec{u}(V)=(S+I) \vec{u}^{\prime}(V)$, then it is also possible to identify the kind of coupling it represents.

For the ET-matrix defined above, let $\left|s_{i i}\right|=r, i=1, \ldots, n$ and $\left|s_{i j}\right|=\left|s_{l m}\right|=t, i \neq j$, $l \neq m, i, j, l, m=1, \ldots, n$. The matrices have the representation given by

$$
S=D_{\theta}^{*} C D_{\theta}
$$

where

$$
\begin{aligned}
C & =\left(\begin{array}{ccccc}
r & t & t & \cdots & t \\
t & r & t a_{23} & \cdots & t a_{2 n} \\
t & t \bar{a}_{23} & r & & t a_{3 n} \\
\vdots & \vdots & & \ddots & \vdots \\
t & t \bar{a}_{2 n} & t \bar{a}_{3 n} & \cdots & r
\end{array}\right), \\
D_{\theta} & =\operatorname{diag}\left\{e^{i \theta_{1}}, \ldots, e^{i \theta_{n}}\right\}, \quad \theta_{i} \in[-\pi, \pi], \quad i=1,2, \ldots, n-1, \\
a_{i j} & \in\left\{\mathbb{C}:\left|a_{i j}\right|=1, \text { for all } i=2, \ldots, n-1, j=3, \ldots, n\right\} .
\end{aligned}
$$

It is easy to see that the matrix $C$ has the property that $\left|c_{i j}\right|=\left|s_{i j}\right|, i, j=1,2, \ldots, n$. Therefore it suffices in studying the equi-transmitting properties of $S$ to consider the matrix $C$. The two matrices are equal up to the phases described by the matrix $D_{\theta}$.

Having an ET-matrix $S$ one can obtain another ET-matrix by means of appropriate matrix permutation. Below we discuss such transformations

1. Let $D$ be a diagonal matrix whose diagonal entries comprise \pm 1 . Then the transformation 


$$
S_{D}=D^{-1} S D
$$

has the effect that certain entries of $S$ have their signs reversed. However signs of the diagonal entries remain unchanged because the reversal of their signs is done. Such a transformation yields yet another ET-matrix since $\left|s_{D_{i j}}\right|=\left|s_{i j}\right|$, $i, j=1,2, \ldots, n$.

2. The transformation $S \mapsto-S$ has the effect of reversing the signs of all the entries of $S$. It is clear that the resulting matrix is again an ET-matrix.

3. Let $P_{l m}$ be a permutation matrix. The matrix

$$
S_{P}=P_{l m} S P_{l m}
$$

is obtained by interchanging rows and columns $i$ and $j$. Therefore $\left|s_{P_{i j}}\right|=\left|s_{i j}\right|$, $i, j=1,2, \ldots, n$ meaning that $S_{P}$ is also an ET-matrix.

From the foregoing it can be seen that the study of ET-matrices can be done up to the above transformations.

\section{Possible values of $r$ and $t$}

From the definition of the ET-matrix, the numbers $r$ and $t$ are nonnegative. Since the matrix $C$ is unitary, the rows form normalised vectors. Thus row 1 yields the equation

$$
r^{2}+(n-1) t^{2}=1
$$

which is key to the construction of matrix $C$. From the equation and the condition that $r$ and $t$ are nonnegative, it follows that $r, t \in[0,1]$.

When $r=1$, then $t=0$ and from the representation of $C$ (see equation (2.1)) it can be seen that the resulting matrix is a diagonal matrix whose diagonal entries comprises \pm 1 . This is a trivial class of ET-matrices. On the other hand when $r=0$ then $t=1$ and one obtains a RET-matrix. These have been discussed up to order six in [16].

Since $S$ is Hermitian and unitary its spectrum consists of \pm 1 . Let $n^{+}$be the multiplicity of +1 in the spectrum of $S$. Let $v^{+}$be the number of positive diagonal entries of the matrix $S$. Then if the order of $S$ is $n$, the numbers $n^{+}$and $v^{+}$can assume any value in the set $\{0,1, \ldots, n\}$. The trace of $S$ is given by the relations $\operatorname{Tr}(S)=2 n^{+}-n=r\left(2 v^{+}-n\right)$. This implies that

$$
r=\frac{2 n^{+}-n}{2 v^{+}-n}
$$

This formula is applicable if and only if $2 v^{+}-n \neq 0$. Assuming this condition is taken into consideration, it follows that $r=1$ if and only if $n^{+}=v^{+}$, while $r=0$ if and only if $n^{+}=\frac{1}{2} n$.

It should be noted that cases when $2 v^{+}-n=0$, which implies that $v^{+}=\frac{1}{2} n$, only arise when $n$ is even. In such a case the formula (3.2) cannot be used to determine the value of $r$. The only option in determining $r$ and hence constructing $S$, if possible, is by using the definition of $S$ and its corresponding representation. This case has turned out to be significant despite our initial skepticism of what can be obtained from it. It is only in this case that an ET-matrix other than those generated by the standard matching condition matrix can be constructed.

The figures below give a graphical depiction of the possible values of $r$. The first figure is for the case when $n$ is odd, while the second figure is for the case when $n$ is even.

The diagonal white cells represent cases where $r=1$ and for which the corresponding ET-matrix is a diagonal matrix. The orange cells represent cases where $r>1$ and hence no ET-matrix can be constructed. The blue cells represent cases where $r<0$ so that no 


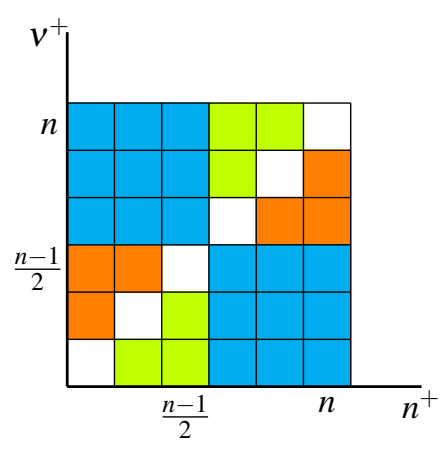

(a)

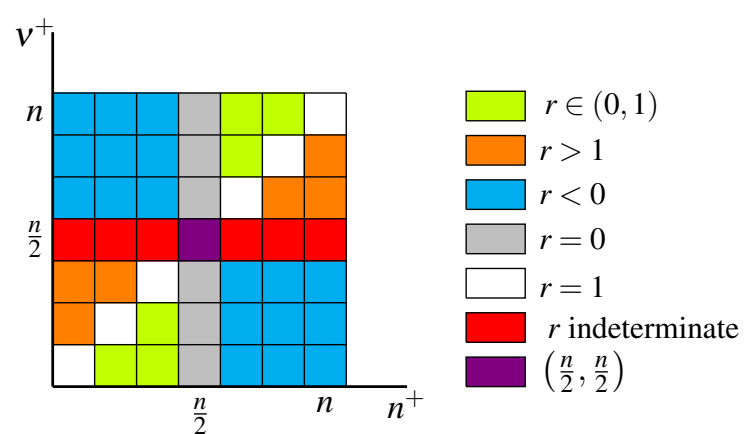

(b)

Figure 1. Possible values of r; (a) when $n$ is odd and (b) when $n$ is even

ET-matrix can be constructed. The lime cells represent cases where $r \in(0,1)$ and where there is the possibility of the existence of ET-matrices. In figure (b), the light gray cells represent cases where $r=0$ and where the corresponding ET-matrices are reflectionless. The red cells in figure (b) represent where $r$ is not defined so that no ET-matrix can be constructed. In the same figure, the middle violet cell represent where $r$ is indeterminate from formula (3.2) but where it is possible to construct ET-matrices from the definition of $S$.

The various cases to be considered when investigating the existence of ET-matrices will be denoted by the ordered pair $\left(n^{+}, v^{+}\right)$. It should be noted that the case $\left(n-n^{+}, n-v^{+}\right)$yields the same values of $r$ and $t$.

We now discuss the viable cases for the existence of an ET-matrix when $r, t \in(0,1)$. The cases where $n^{+}=v^{+}$are excluded since they give rise to $r=1$. The exception to this is the case $\left(n^{+}, v^{+}\right)=\left(\frac{n}{2}, \frac{n}{2}\right)$ when $n$ is even because it is also viable for the existence of the required ET-matrix. When $n$ is odd then cases for which $r, t \in(0,1)$ are given by

$$
\left(n^{+}, v^{+}\right) \text {and }\left(n-n^{+}, n-v^{+}\right), \quad \text { where } \quad\left\{\begin{array}{l}
n^{+} \in\left\{1,2, \ldots, \frac{n-1}{2}\right\} \\
v^{+} \in\left\{0,1 \ldots, \frac{n-3}{2}\right\} .
\end{array}\right.
$$

On the other hand when $n$ is even then such cases are given by

$$
\left(n^{+}, v^{+}\right),\left(n-n^{+}, n-v^{+}\right) \text {and }\left(\frac{n}{2}, \frac{n}{2}\right), \text { where }\left\{\begin{array}{l}
n^{+} \in\left\{1,2, \ldots, \frac{n}{2}-1\right\} \\
v^{+} \in\left\{0,1 \ldots, \frac{n}{2}-2\right\}
\end{array}\right.
$$

\section{Important general examples}

\section{Diagonal matrices}

For all $n$, whenever $r=1$, there exists an equi-transmitting diagonal matrix. This matrix is not interesting in the framework of quantum graphs since it describes completely independent (not connected) edges. Such matrices will be called trivial ET-matrices, while those corresponding to $r \in(0,1)$ will be called non trivial ET-matrices.

\section{Reflectionless equi-transmitting matrices}

These are obtained when $r=0$. We have discussed these matrices in [16] where it has been proved that such matrices exist only when $n$ is even. 


\section{Standard matching conditions equi-transmitting matrices}

This is a special class of ET-matrices for $r \in(0,1)$ and it appears when we impose SMC. For every incoming wave in a star graph with $n$ infinite edges there are both reflected waves along the same edge and transmitted waves along the other $n-1$ edges. This condition is described by the equation

$$
u(x)=\left\{\begin{array}{l}
e^{-i k x}+R_{i} e^{i k x}, x \in E_{i} \\
T_{j i} e^{i k x}, x \in E_{j}
\end{array}\right.
$$

where $R_{i}$ is the reflection coefficient along edge $E_{i}$ and $T_{j i}$ is the transmission coefficient of waves transmitted from edge $E_{i}$ into edge $E_{j}$.

Suppose the coupling of the edges at the central vertex $V$ is described by the SMC. Suppose also that the edges are parameterized as $E_{j}=[0, \infty), j=1, \ldots, n$. These conditions are invariant under permutations. Therefore all reflection coefficients $R_{i}$ are equal and all transmission coefficients $T_{j i}$ are also equal. The requirement that $u_{j}(0)=u_{l}(0), j, l=1 \ldots, n$ together with equation (4.1) yield the equation $R+1=T$. On the other hand the conditions $\sum \partial_{n} u(0)=0$ as well as equation (4.1) result in the equation $i k(R-1+(n-1) T)=0$. These two equations yield the values $T=\frac{2}{n}$ and $R=-1+\frac{2}{n}$. Thus the matrix $S$ arising from the SMC is given by

$$
s_{j l}= \begin{cases}-1+\frac{2}{n}, & j=l \\ \frac{2}{n}, & j \neq l .\end{cases}
$$

It is easy to see that this matrix is Hermatian (symmetric in this case since all the entries are real). It is also easy to see that $S^{t} S=S S^{t}=I$, i.e. $S$ is orthogonal. Thus $S$ is Hermitian, unitary and equi-transmitting for all $n \geq 2$. Using the notations introduced in section 2 , we see that

$$
\left|s_{j l}\right|=\left\{\begin{array}{ll}
r=\left|-1+\frac{2}{n}\right|, & j=l \\
t=\frac{2}{n}, & j \neq l
\end{array} .\right.
$$

This construction determines a Hermitian unitary equi-transmitting matrix $S$ of order $n$ for all $n \geq 3$ and for $r \in(0,1)$. We will refer to this matrix as the SMC-matrix.

Cases $(1,0)$ and $(n-1, n)$

For any given $n$ the case $\left(n^{+}, v^{+}\right)=(1,0)$ yields the equation $-n r=-(n-2)$ which implies that $r=1-\frac{2}{n}$. When this value of $r$ is substituted into the equation $r^{2}+(n-1) t^{2}=1$ and the positive value of $t$ evaluated, it is obtained as $t=\frac{2}{n}$. Since in this case all the diagonal entries are negative, the associated matrix $C$ has the representation

$$
C_{1,0}=\left(\begin{array}{ccccc}
-1+\frac{2}{n} & \frac{2}{n} & \frac{2}{n} & \cdots & \frac{2}{n} \\
\frac{2}{n} & -1+\frac{2}{n} & \frac{2}{n} a_{23} & \cdots & \frac{2}{n} a_{2 n} \\
\frac{2}{n} & \frac{2}{n} \bar{a}_{23} & -1+\frac{2}{n} & & \frac{2}{n} a_{3 n} \\
\vdots & \vdots & & \ddots & \\
\frac{2}{n} & \frac{2}{n} \bar{a}_{2 n} & \frac{2}{n} \bar{a}_{3 n} & \cdots & -1+\frac{2}{n}
\end{array}\right)
$$

where $a_{i j} \in\{z \in \mathbb{C}:|z|=1, i=2, \ldots, n-1, j=3, \ldots, n, i<j\}$. Thus there are $\frac{1}{2}(n-1)$ $(n-2)$ such parameters. The inner products of the rows yield certain $\frac{1}{2} n(n-1)$ equations since the rows are orthogonal. The orthogonality between the first row and all the other rows give the following $n-1$ equations 


$$
\begin{aligned}
2 r+t\left(a_{23}+a_{24}+\cdots+a_{2, n-1}+a_{2 n}\right) & =0 \\
2 r+t\left(\bar{a}_{23}+a_{34}+\cdots+a_{3, n-1}+a_{3 n}\right) & =0 \\
\vdots & \\
2 r+t\left(\bar{a}_{2, n-1}+\bar{a}_{3, n-1}+\cdots+\bar{a}_{n-2, n-1}+a_{n-1, n}\right) & =0 \\
2 r+t\left(\bar{a}_{2 n}+\bar{a}_{3 n}+\cdots+\bar{a}_{n-2, n}+\bar{a}_{n-1, n}\right) & =0
\end{aligned}
$$

These equations can be rewritten as follows

$$
\begin{aligned}
a_{23}+a_{24}+\cdots+a_{2, n-1}+a_{2 n} & =\frac{2 r}{t} \\
\bar{a}_{23}+a_{34}+\cdots+a_{3, n-1}+a_{3 n} & =\frac{2 r}{t} \\
\vdots & \\
\bar{a}_{2, n-1}+\bar{a}_{3, n-1}+\cdots+\bar{a}_{n-2, n-1}+a_{n-1, n} & =\frac{2 r}{t} \\
\bar{a}_{2 n}+\bar{a}_{3 n}+\cdots+\bar{a}_{n-2, n}+\bar{a}_{n-1, n} & =\frac{2 r}{t} .
\end{aligned}
$$

Each of the above equations has $n-2$ parameters while on the right hand side we have that $\frac{2 r}{t}=2\left(1-\frac{2}{n}\right) \frac{n}{2}=n-2$. Since each parameter is unimodular, all the equations are satisfied if and only if each of the parameters is unity, i.e. $a_{i j}=1, i=2, \ldots, n-1, j=$ $3, \ldots, n, i<j$. All the other $\frac{1}{2}(n-2)(n-1)$ equations are of the form

$$
-2 r \bar{a}_{i j}+t\left(1+\sum_{l=3}^{n} a_{i l} a_{j l}\right)=0, \quad i=2, \ldots, n-1, j=3, \ldots, n, i \neq l \neq j, i<j .
$$

The terms under the summations could involve the conjugates of the parameters. All these equations are also satisfied when all the parameters are unity. It follows that the matrix determined is precisely the smc matrix and is given as follows

$$
C_{1,0}=\left(\begin{array}{ccccc}
-1+\frac{2}{n} & \frac{2}{n} & \frac{2}{n} & \cdots & \frac{2}{n} \\
\frac{2}{n} & -1+\frac{2}{n} & \frac{2}{n} & \cdots & \frac{2}{n} \\
\frac{2}{n} & \frac{2}{n} & -1+\frac{2}{n} & & \frac{2}{n} \\
\vdots & \vdots & & \ddots & \vdots \\
\frac{2}{n} & \frac{2}{n} & \frac{2}{n} & \cdots & -1+\frac{2}{n}
\end{array}\right) .
$$

The case $\left(n^{+}, v^{+}\right)=(n-1, n)$ can be analysed in a similar way leading to the matrix

$$
C_{n-1, n}=\left(\begin{array}{ccccc}
1-\frac{2}{n} & \frac{2}{n} & \frac{2}{n} & \cdots & \frac{2}{n} \\
\frac{2}{n} & 1-\frac{2}{n} & -\frac{2}{n} & \cdots & -\frac{2}{n} \\
\frac{2}{n} & -\frac{2}{n} & 1-\frac{2}{n} & & -\frac{2}{n} \\
\vdots & \vdots & & \ddots & \vdots \\
\frac{2}{n} & -\frac{2}{n} & -\frac{2}{n} & \cdots & 1-\frac{2}{n}
\end{array}\right) .
$$

The relation between the two matrices is given by

$$
C_{n-1, n}=-D C_{1,0} D
$$


where $D$ is an $n \times n$ diagonal matrix given by $D=\operatorname{diag}\{-1,1, \ldots, 1\}$. This means that for all $n \geq 2$, the cases $(1,0)$ and $(n-2, n)$ give the same transition probabilities as the SMC.

\section{Equi-transmitting matrices of order 2,3,4 and 5.}

This section is devoted to the discussion of nontrivial ET-matrices of orders 2,3,4 and 5 for the values of $r \in(0,1)$. The cases $(1,0)$ and $(n-1, n)$ have been covered and so whenever they arise, we will simply state the results. All the other cases are appropriately discussed.

\section{Order 2}

The only case leading to $r \in(0,1)$ is when the trace of the matrix is zero. This is given by $\left(n^{+}, v^{+}\right)=(1,1)$. The corresponding representation of matrix $C$ is

$$
C=\left(\begin{array}{cc}
r & t \\
t & -r
\end{array}\right)
$$

Normalisation of the rows yield the relation $t=\sqrt{1-r^{2}}$ where $r \in(0,1)$. The corresponding ET-matrices $C$ are a one-parameter family given as follows

$$
C_{1,1}=\left(\begin{array}{cc}
r & \sqrt{1-r^{2}} \\
\sqrt{1-r^{2}} & -r
\end{array}\right)
$$

Observe that the matrix has zero trace and it is a one-parameter family of matrices.

\section{Order 3}

When $n=3$, then the only cases yielding $r$ and $t$ in the interval $(0,1)$ are given by the ordered pairs $(1,0)$ and $(2,3)$. These have been covered in the previous section. The matrices $C$ corresponding to these cases are respectively given as

$$
C_{1,0}=\frac{1}{3}\left(\begin{array}{ccc}
-1 & 2 & 2 \\
2 & -1 & 2 \\
2 & 2 & -1
\end{array}\right) \quad \text { and } \quad C_{2,3}=\frac{1}{3}\left(\begin{array}{ccc}
1 & 2 & 2 \\
2 & 1 & -2 \\
2 & -2 & 1
\end{array}\right)
$$

From equation (3.3) we see that any other case will not yield values $r$ and $t$ in the interval $(0,1)$. Thus when $n=3$, then the only nontrivial ET-matrices are those generated by the SMC-matrix.

\section{Order 4}

From (3.4) we that the cases to consider when $n=4$ in order to obtain the values of $r$ and $t$ in the interval $(0,1)$ are $(1,0),(3,4)$ and $(2,2)$.

1. Cases $(1,0)$ and $(3,4)$ The corresponding matrices are equivalent to the standard matching conditions matrix as discussed in the previous section. The respective $C$ matrices are

$$
C_{1,0}=\frac{1}{2}\left(\begin{array}{cccc}
-1 & 1 & 1 & 1 \\
1 & -1 & 1 & 1 \\
1 & 1 & -1 & 1 \\
1 & 1 & 1 & -1
\end{array}\right) \quad \text { and } \quad C_{3,4}=\frac{1}{2}\left(\begin{array}{cccc}
1 & 1 & 1 & 1 \\
1 & 1 & -1 & -1 \\
1 & -1 & 1 & -1 \\
1 & -1 & -1 & 1
\end{array}\right)
$$

3. Case $(2,2)$ In this case the trace is zero and $r$ cannot be determined from the formula (3.2) but it is in the interval $(0,1)$. The number of positive and negative diagonal entries are equal and the corresponding matrix $C$ can be represented by 


$$
C=\left(\begin{array}{cccc}
r & t & t & t \\
t & r & a t & b t \\
t & \bar{a} t & -r & c t \\
t & \bar{b} t & \bar{c} t & -r
\end{array}\right)
$$

where $a, b, c \in\{z \in \mathbb{C}:|z|=1\}$. Using the orthogonality of the rows we have the following system of equations

$$
\begin{aligned}
& 2 r+t(a+b)=0 \\
& \bar{a}+c=0 \\
& \bar{b}+\bar{c}=0 \\
& 1+\bar{b} c=0 \\
& 1+\overline{a c}=0 \\
& -2 r \bar{c}+t(1+a \bar{b})=0
\end{aligned}
$$

Solving for the parameters $a, b, c$, it is obtained that $a$ can be chosen arbitrary, in which case $b=\bar{a}$ and $c=-\bar{a}$. A relation between $r$ and $t$ based on the system is obtained as $r+t \mathfrak{R}(a)=0$. Using this relation between $r$ and $t$ and that in (3.1) one obtains that $t=\frac{1}{\sqrt{3+\Re(a)^{2}}}$ and $r=\frac{-\Re(a)}{\sqrt{3+\Re(a)^{2}}}$. The parameter $a$ is chosen such that $\mathfrak{R}(a)<0$ because $r>0$. For example suppose $a=-e^{i \theta}$, then the appropriate values of $\theta$ are in the interval $\left(-\frac{\pi}{2}, \frac{\pi}{2}\right)$. The corresponding matrix $C$ is given as follows

$$
C_{2,2}=\frac{1}{\sqrt{3+\cos ^{2} \theta}}\left(\begin{array}{cccc}
\cos \theta & 1 & 1 & 1 \\
1 & \cos \theta & -e^{i \theta} & -e^{-i \theta} \\
1 & -e^{-i \theta} & -\cos \theta & e^{-i \theta} \\
1 & -e^{i \theta} & e^{i \theta} & -\cos \theta
\end{array}\right)
$$

Therefore the matrix $C$ is given by

$$
C_{2,2}=\frac{1}{\sqrt{3+\Re(a)^{2}}}\left(\begin{array}{cccc}
-\Re(a) & 1 & 1 & 1 \\
1 & -\Re(a) & a & \bar{a} \\
1 & \bar{a} & \Re(a) & -\bar{a} \\
1 & a & -a & \Re(a)
\end{array}\right)
$$

It is therefore observed that when $n=4$, all nontrivial ET-matrices obtained can be put into the following two categories

1. Matrices generated by the SMC-matrix.

2. One parameter family matrices $C$ with zero trace.

\section{Order 5}

When $n=5$, we establish that the only nontrivial ET-matrices are those generated by the SMCmatrix. According to (3.3) the cases that give rise to $r, t \in(0,1)$ when $n=5$ are $(1,0)$, $(4,5),(2,0),(3,5),(2,1)$ and $(3,4)$. Each case is discussed in the sequel.

1. Cases $(1,0)$ and $(4,5)$. The respective matrices $C$ are

$$
C_{1,0}=\frac{1}{5}\left(\begin{array}{ccccc}
-3 & 2 & 2 & 2 & 2 \\
2 & -3 & 2 & 2 & 2 \\
2 & 2 & -3 & 2 & 2 \\
2 & 2 & 2 & -3 & 2 \\
2 & 2 & 2 & 2 & -3
\end{array}\right) \quad \text { and } \quad C_{4,5}=\frac{1}{5}\left(\begin{array}{ccccc}
3 & 2 & 2 & 2 & 2 \\
2 & 3 & -2 & -2 & -2 \\
2 & -2 & 3 & -2 & -2 \\
2 & -2 & -2 & 3 & -2 \\
2 & -2 & -2 & -2 & 3
\end{array}\right)
$$

The first matrix is precisely the SMC-matrix, while the second is generated by it in the sense of the transformation discussed in section 4. 
3. Case $(2,0)$. In this case all diagonal entries are negative and two eigenvalues are positive. The values $r$ and $t$ are $\frac{1}{5}$ and $\frac{\sqrt{6}}{5}$ respectively. The corresponding matrix $C$ is represented as follows

$$
C=\left(\begin{array}{ccccc}
-r & t & t & t & t \\
t & -r & a t & b t & c t \\
t & \bar{a} t & -r & d t & e t \\
t & \bar{b} t & \bar{d} t & -r & f t \\
t & \bar{c} t & \bar{e} t & \bar{f} t & -r
\end{array}\right)
$$

where $a, \ldots, f \in\{z \in \mathbb{C}:|z|=1\}$. Since the rows of $C$ are orthogonal, their inner products yield the following system of equations

$$
\begin{aligned}
& \left\{\begin{array} { l l } 
{ - 2 r + t ( a + b + c ) = 0 } & { ( 1 ) } \\
{ - 2 r + t ( \overline { a } + d + e ) = 0 } & { ( 2 ) } \\
{ - 2 r + t ( \overline { b } + \overline { d } + f ) = 0 } & { ( 3 ) } \\
{ - 2 r + t ( \overline { c } + \overline { e } + \overline { f } ) = 0 } & { ( 4 ) }
\end{array} \quad \left\{\begin{array}{l}
-2 \bar{a} r+t(1+\bar{b} d+\bar{c} e)=0 \\
-2 \bar{b} r+t(1+\bar{a} \bar{d}+\bar{c} f)=0 \\
-2 \bar{c} r+t(1+\overline{a e}+\overline{b \bar{f}})=0
\end{array}\right.\right. \\
& \left\{\begin{array}{l}
-2 \bar{d} r+t(1+a \bar{b}+\bar{e} f)=0 \\
-2 \bar{e} r+t(1+a \bar{c}+\overline{d f})=0
\end{array}\right.
\end{aligned}
$$

Equations (2) to (4) can be written as

$$
M 1=-\vec{X}
$$

where

$$
\begin{aligned}
& M=\left(\begin{array}{ccc}
-\alpha & \bar{d} & \bar{e} \\
d & -\alpha & \bar{f} \\
e & f & -\alpha
\end{array}\right) \\
& \mathbf{1}=[1,1,1]^{t}, \quad \vec{X}=[a, b, c]^{t}, \quad \alpha=\frac{2 r}{t}=\frac{\sqrt{6}}{3}
\end{aligned}
$$

Equations (5) to (7) can be written as

$$
M \vec{X}=-\mathbf{1} .
$$

Substituting the value of $\vec{X}$ from (5.2) into (5.4) yields that

$$
M^{2} \mathbf{1}=\mathbf{1} .
$$

The matrix $M$ is Hermitian and so it possesses a complete orthonormal set of eigenvectors. Now suppose that $\lambda$ is an eigenvalue for $M$ corresponding to the eigenvector $\psi$ then

$$
M \vec{\psi}=\lambda \vec{\psi}, \quad \Longleftrightarrow \quad M^{2} \vec{\psi}=\lambda^{2} \vec{\psi}
$$

Comparing the second equation in (5.6) and equation (5.5) it can be seen that $\lambda^{2}=1$ is the eigenvalue for $M^{2}$ corresponding to the eigenvector $\mathbf{1}$ and it follows that

$$
M 1= \pm 1 .
$$

Comparing (5.7) and (5.2) we obtain that

$$
\vec{X}= \pm \mathbf{1}
$$


Therefore either $a=b=c=1$ or $a=b=c=-1$. The substitution of the values into equation (1) in (5.1) yields the contradictions that $\pm 3=\frac{\sqrt{6}}{3}$. Thus the case $(2,0)$ does not yield an ET-matrix for $r, t \in(0,1)$.

4. Case $(3,5)$. This case is completely analogous to case $(2,0)$ and similar contradictions are obtained hence there is no ET-matrix.

5. Case $(2,1)$. In this case one diagonal entry is positive and two eigenvalues are positive.This implies that $r=\frac{1}{3}$ and $t=\frac{\sqrt{2}}{3}$. The matrix associated to this case can be written as

$$
C=\left(\begin{array}{ccccc}
r & t & t & t & t \\
t & -r & a t & b t & c t \\
t & \bar{a} t & -r & d t & e t \\
t & \bar{b} t & \bar{d} t & -r & f t \\
t & \bar{c} t & \bar{e} t & \bar{f} t & -r
\end{array}\right)
$$

where $a, \ldots, f \in\{z \in \mathbb{C}:|z|=1\}$. Since the rows of $C$ are orthogonal, their inner products yield the following system of equations

$$
\begin{aligned}
& \left\{\begin{array}{l}
a+b+c=0 \\
\bar{a}+d+e=0 \\
\bar{b}+\bar{d}+f=0 \\
\bar{c}+\bar{e}+\bar{f}=0
\end{array}\right. \\
& \left\{\begin{array}{ll}
-2 \bar{d} r+t(1+a \bar{b}+\bar{e} f)=0 \\
-2 \bar{e} r+t(1+a \bar{c}+\overline{d f})=0
\end{array} \quad(9) \quad\{-2 \bar{f} r+t(1+b \bar{c}+d \bar{e})=0\right.
\end{aligned}
$$

Equatiions (2) to (4) can be written as

$$
A \mathbf{1}=-\vec{X}
$$

where

$$
A=\left(\begin{array}{lll}
0 & \bar{d} & \bar{e} \\
d & 0 & \bar{f} \\
e & f & 0
\end{array}\right)
$$

and vectors 1 and $\vec{X}$ are as given in (5.3). Using the same notations, equations (5) to $(7)$ can also be written as

$$
(A-\alpha I) \vec{X}=-\mathbf{1}
$$

where $\alpha=\frac{2 r}{t}=\sqrt{2}$. Substituting the value of $\vec{X}$ from (5.9) into (5.10) yields that

$$
\left(A^{2}-\alpha A-I\right) \mathbf{1}=\mathbf{0} \text {. }
$$

The matrix $A$ is Hermitian and thus has a complete set of eigenvectors which can be chosen orthonormal. Therefore if $\lambda$ is an eigenvalue for $A$ corresponding to the eigenvector $\psi$ then

$$
A \vec{\psi}=\lambda \vec{\psi}, \quad \Leftrightarrow \quad\left(A^{2}-\alpha A-I\right) \vec{\psi}=\left(\lambda^{2}-\alpha \lambda-1\right) \vec{\psi} .
$$

From equations (5.11) and (5.12) it follows that 1 belongs to the kernel of $A^{2}-$ $\alpha A-I$ if and only if it is the eigenvector for $A$ corresponding to the eigenvalue $\lambda$ satisfying $\lambda^{2}-\alpha \lambda-1=0$. Therefore 


$$
\lambda=\lambda_{ \pm}=\frac{\sqrt{2} \pm \sqrt{6}}{2} \neq 0
$$

and

$$
A \mathbf{1}=\lambda_{ \pm} \mathbf{1}
$$

Comparing the equations (5.9) and (5.13) it can be seen that

$$
\vec{X}=-\lambda_{ \pm} \mathbf{1}
$$

Therefore either $a=b=c=-\lambda_{+}$or $a=b=c=-\lambda_{-}$. Substituting these values into equation (1) in (5.8) one obtains that $-3 \lambda_{ \pm}=\sqrt{2}$ which are contradictions. Thus the case $(2,1)$ does not yield any nontrivial ET-matrix.

6. Case $(3,4)$. The values of $r$ and $t$ are obtained as $\frac{1}{3}$ and $\frac{\sqrt{2}}{3}$ respectively. Only one of the diagonal entries is negative and so the associated matrix $C$ can be written as follows

$$
C=\left(\begin{array}{ccccc}
r & t & t & t & t \\
t & r & a t & b t & c t \\
t & \bar{a} t & r & d t & e t \\
t & \bar{b} t & \bar{d} t & r & f t \\
t & \bar{c} t & \bar{e} t & \bar{f} t & -r
\end{array}\right)
$$

where $a, \ldots, f \in\{z \in \mathbb{C}:|z|=1\}$. From the orthogonality of the rows of $S$ we obtain the following system of equations

$$
\begin{gathered}
\left\{\begin{array} { l l } 
{ 2 r + t ( a + b + c ) = 0 } & { ( 1 ) } \\
{ 2 r + t ( \overline { a } + d + e ) = 0 } & { ( 2 ) } \\
{ 2 r + t ( \overline { b } + \overline { d } + f ) = 0 } & { ( 3 ) } \\
{ \overline { c } + \overline { e } + \overline { f } = 0 } & { ( 4 ) }
\end{array} \quad \left\{\begin{array}{l}
2 \bar{a} r+t(1+\bar{b} d+\bar{c} e)=0 \\
2 \bar{b} r+t(1+\bar{a} \bar{d}+\bar{c} f)=0 \\
1+\overline{a e}+\overline{b f}=0
\end{array}\right.\right. \\
\left\{\begin{array}{ll}
2 \bar{d} r+t(1+a \bar{b}+\bar{e} f)=0 & (8) \\
1+a \bar{c}+\overline{d f}=0 & (9)
\end{array} \quad\{1+b \bar{c}+d \bar{e}=0\right.
\end{gathered}
$$

This case is similar to case $(2,1)$ and can be analysed in a similar fashion with the following modifications

1. From equations (1) to (3) matrix $A$ and vector $\vec{X}$ are obtained respectively as

$$
\left(\begin{array}{ccc}
0 & a & b \\
\bar{a} & 0 & d \\
\bar{b} & \bar{d} & 0
\end{array}\right) \quad \text { and } \quad\left(\begin{array}{c}
c \\
e \\
f
\end{array}\right)
$$

2. Equations (7), (9) and (10) yield the matrix equation $A \vec{X}=-\mathbf{1}$.

The analysis shows that

$$
\vec{X}=-\frac{1}{\lambda_{ \pm}} \mathbf{1}, \quad \lambda_{ \pm}=\frac{-\sqrt{2} \pm \sqrt{6}}{2} .
$$

Substituting the corresponding values of the parameters into equation (4) of the system (5.14) yields the contradiction that $-3 \frac{1}{\lambda_{ \pm}}=0$. Thus no nontrivial ET-matix exists in this case as well. 
From all the cases considered, nontrivial ET-matrices have been constructed only in the cases $(1,0)$ and $(4,5)$. As has been stated above, these cases are generated by the SMC-matrix. No nontrivial ET-matrix can be obtained in all the other cases.

\section{Equi-transmitting matrices of order 6}

When $n=6$, then (3.4) shows that the cases for which $r, t \in(0,1)$ are

$$
(1,0), \quad(5,6), \quad(2,0), \quad(4,6), \quad(2,1), \quad(4,5) \text { and }(3,3) .
$$

The cases $(1,0)$ and $(5,6)$ have already been covered in 4 and so here we simply state the results. The discussion of each of the other cases begins with a matrix representation in terms of some ten unimodular parameters so that $C$ is an ET-matrix. The parameters are then determined so as to obtain an explicit form of the matrix. By construction all the rows and columns are normalised. Therefore $C$ is unitary if and only if the rows are orthogonal, which gives us a system of 15 equations. The system is then reduced until either an explicit form of the matrix determined or a contradiction is obtained. In determining the parameters, the following lemma is applied to appropriate equations ${ }^{1}$. Whenever the matrix is obtained, it may be completely determined or it may be given in terms of some arbitrary unimodular parameters.

Lemma 1. The sum of four complex numbers $z_{1}, z_{2}, z_{3}, z_{4}$ of equal magnitude equals zero if and only if at least one of the following cases occur:

$$
\begin{gathered}
z_{2}=-z_{1}, z_{4}=-z_{3}, \\
\text { or } \\
z_{3}=-z_{1}, z_{4}=-z_{2}, \\
\text { or } \\
z_{4}=-z_{1}, z_{3}=-z_{2} .
\end{gathered}
$$

Now we consider each of the cases listed in (6.1):

1. Cases $(1,0)$ and $(5,6)$. These cases have been discussed in section 4 and the respective matrices $C$ given below are generated by the SMC-matrix (see section $4)$.

$C_{1,0}=\frac{1}{3}\left(\begin{array}{cccccc}-2 & 1 & 1 & 1 & 1 & 1 \\ 1 & -2 & 1 & 1 & 1 & 1 \\ 1 & 1 & -2 & 1 & 1 & 1 \\ 1 & 1 & 1 & -2 & 1 & 1 \\ 1 & 1 & 1 & 1 & -2 & 1 \\ 1 & 1 & 1 & 1 & 1 & -2\end{array}\right)$ and $C_{5,6}=\frac{1}{3}\left(\begin{array}{cccccc}2 & 1 & 1 & 1 & 1 & 1 \\ 1 & 2 & -1 & -1 & -1 & -1 \\ 1 & -1 & 2 & -1 & -1 & -1 \\ 1 & -1 & -1 & 2 & -1 & -1 \\ 1 & -1 & -1 & -1 & 2 & -1 \\ 1 & -1 & -1 & -1 & -1 & 2\end{array}\right)$

2. Case $(2,0)$. In this case all diagonal entries are negative while two of the eigenvalues are positive. The values of $r$ and $t$ are therefore obtained as $\frac{1}{3}$ and $\frac{2 \sqrt{2}}{3 \sqrt{5}}$ respectively. The associated matrix $C$ is represented as follows

\footnotetext{
${ }^{1}$ Details are in Appendix B 


$$
C=\left(\begin{array}{cccccc}
-r & t & t & t & t & t \\
t & -r & a t & b t & c t & d t \\
t & \bar{a} t & -r & e t & f t & g t \\
t & \bar{b} t & \bar{e} t & -r & h t & l t \\
t & \bar{c} t & \bar{f} t & \bar{h} t & -r & m t \\
t & \bar{d} t & \bar{g} t & \bar{l} t & \bar{m} t & -r
\end{array}\right)
$$

where $a, b, c, d, e, f, g, h, l, m \in\{z \in \mathbb{C}:|z|=1\}$. Since the rows are orthogonal, determining their inner products yield the following system of equations

$$
\begin{gathered}
\left\{\begin{array} { l l } 
{ - 2 r + t ( a + b + c + d ) = 0 } & { ( 1 ) } \\
{ - 2 r + t ( \overline { a } + e + f + g ) = 0 } & { ( 2 ) } \\
{ - 2 r + t ( \overline { b } + \overline { e } + h + l ) = 0 } & { ( 3 ) } \\
{ - 2 r + t ( \overline { c } + \overline { f } + \overline { h } + m ) = 0 } & { ( 4 ) } \\
{ - 2 r + t ( \overline { d } + \overline { g } + \overline { l } + \overline { m } ) = 0 } & { ( 5 ) }
\end{array} \quad \left\{\begin{array}{l}
-2 \bar{a} r+t(1+\bar{b} e+\bar{c} f+\bar{d} g)=0 \\
-2 \bar{b} r+t(1+\overline{a e}+\bar{c} h+\bar{d} l)=0 \\
-2 \bar{c} r+t(1+\bar{a} \bar{f}+\overline{b h}+\bar{d} m)=0 \\
-2 \bar{d} r+t(1+\overline{a g}+\overline{b l}+\overline{c m})=0
\end{array}\right.\right. \\
\left\{\begin{array} { l l } 
{ - 2 \overline { e } r + t ( 1 + a \overline { b } + \overline { f } h + \overline { g } l ) = 0 } \\
{ - 2 \overline { f } r + t ( 1 + a \overline { c } + \overline { e } \overline { h } + \overline { g } m ) = 0 } \\
{ - 2 \overline { g } r + t ( 1 + a \overline { d } + \overline { e } \overline { l } + \overline { f } \overline { m } ) = 0 }
\end{array} \quad \left\{\begin{array}{l}
-2 \bar{h} r+t(1+b \bar{c}+e \bar{f}+\bar{l} m)=0 \\
-2 \bar{l} r+t(1+b \bar{d}+e \bar{g}+\bar{h} \bar{m})=0
\end{array}\right.\right.
\end{gathered}
$$

In what follows we consider equations (6) to (9) as a linear system of equations in $a, b, c$ and $d$. The corresponding matrix equation given by

$$
\left(\begin{array}{cccc}
-\alpha & \bar{e} & \bar{f} & \bar{g} \\
e & -\alpha & \bar{h} & \bar{l} \\
f & h & -\alpha & \bar{m} \\
g & l & m & -\alpha
\end{array}\right)\left(\begin{array}{l}
a \\
b \\
c \\
d
\end{array}\right)=\left(\begin{array}{c}
-1 \\
-1 \\
-1 \\
-1
\end{array}\right)
$$

Let the matrix equation be written as

$$
M \vec{X}=-\mathbf{1},
$$

where $M$ is the coefficients matrix, $\vec{X}$ is the vector of the parameters $a, b, c, d$ and $\mathbf{1}=[1,1,1,1]^{t}$. Using these notations then equations (2) to (5) in (6.2) become

$$
\vec{X}=-M 1 .
$$

Substituting this $\vec{X}$ into the matrix equation (6.4) one obtains the following

$$
M^{2} \mathbf{1}=\mathbf{1} .
$$

The matrix $M$ is Hermitian and so it is diagonalizable and has a complete orthonormal set of eigenvectors. Thus suppose $\lambda$ is an eigenvalue for $M$ corresponding to the eigenvector $\vec{\psi}$, then

if and only if

$$
M \vec{\psi}=\lambda \vec{\psi}
$$

$$
M^{2} \vec{\psi}=\lambda^{2} \vec{\psi} .
$$

This means that $\vec{\psi}$ is an eigenvector for $M$ corresponding to the eigenvalue $\lambda$ if and only if it is an eigenvector for $M^{2}$ corresponding to the eigenvalue $\lambda^{2}$. Comparing equations (6.6) and (6.8) we see that the eigenvector for $M^{2}$ corresponding to the eigenvalue $\lambda^{2}=1$ is $\mathbf{1}$. Thus we obtain that $M \mathbf{1}= \pm \mathbf{1}$. Comparing this equation with (6.4) it follows that $\vec{X}=\mathbf{1}$, i.e. either $a=b=c=d=1$ or $a=b=c=d=$ 
-1 . Subsituting these values in equation (1) of the system (6.2) one obtains that $\pm 4=\frac{\sqrt{5}}{\sqrt{2}}$ which is a contradiction.

It thus follows that in the case $(2,0)$ no ET-matrix exists.

3. Case $(4,6)$. This case can be analyzed in a manner similar to case $(2,0)$ resulting in similar contradictions. Therefore no ET-matrix exists here as well.

4. Case $(2,1)$. Only one diagonal entry is positive and two eigenvalues are positive. It is easy to see that this yields the values of $r$ and $t$ to be $\frac{1}{2}$ and $\frac{\sqrt{3}}{2 \sqrt{5}}$. The matrix $C$ corresponding to this case has the following representation

$$
C=\left(\begin{array}{cccccc}
r & t & t & t & t & t \\
t & -r & a t & b t & c t & d t \\
t & \bar{a} t & -r & e t & f t & g t \\
t & \bar{b} t & \bar{e} t & -r & h t & l t \\
t & \bar{c} t & \bar{f} t & \bar{h} t & -r & m t \\
t & \bar{d} t & \bar{g} t & \bar{l} t & \bar{m} t & -r
\end{array}\right)
$$

where $a, b, c, d, e, f, g, h, l, m \in\{z \in \mathbb{C}:|z|=1\}$. From the orthogonality of the rows of $C$ we obtain the following system of equations

$$
\begin{gathered}
\left\{\begin{array} { l l } 
{ a + b + c + d = 0 } & { ( 1 ) } \\
{ \overline { a } + e + f + g = 0 } & { ( 2 ) } \\
{ \overline { b } + \overline { e } + h + l = 0 } & { ( 3 ) } \\
{ \overline { c } + \overline { f } + \overline { h } + m = 0 } & { ( 4 ) } \\
{ \overline { d } + \overline { g } + \overline { l } + \overline { m } = 0 } & { ( 5 ) }
\end{array} \quad \left\{\begin{array}{l}
-2 \bar{a} r+t(1+\bar{b} e+\bar{c} f+\bar{d} g)=0 \\
-2 \bar{b} r+t(1+\overline{a e}+\bar{c} h+\bar{d} l)=0 \\
-2 \bar{c} r+t(1+\bar{a} \bar{f}+\overline{b h}+\bar{d} m)=0 \\
-2 \bar{d} r+t(1+\overline{a g}+\overline{b l}+\overline{c m})=0
\end{array}\right.\right. \\
\left\{\begin{array} { l l } 
{ - 2 \overline { e } r + t ( 1 + a \overline { b } + \overline { f } h + \overline { g } l ) = 0 } & { ( 1 0 ) } \\
{ - 2 \overline { f } r + t ( 1 + a \overline { c } + \overline { e } \overline { h } + \overline { g } m ) = 0 } & { ( 1 1 ) } \\
{ - 2 \overline { g } r + t ( 1 + a \overline { d } + \overline { e } \overline { l } + \overline { f } \overline { m } ) = 0 } & { ( 1 2 ) }
\end{array} \quad \left\{\begin{array}{l}
-2 \bar{h} r+t(1+b \bar{c}+e \bar{f}+\bar{l} m)=0 \\
-2 \bar{l} r+t(1+b \bar{d}+e \bar{g}+\bar{h} \bar{m})=0
\end{array}\right.\right.
\end{gathered}
$$

Equations (6) to (9) can be written as follows

$$
(A-\alpha I) \vec{X}=-\mathbf{1}
$$

where

$$
A=\left(\begin{array}{cccc}
0 & \bar{e} & \bar{f} & \bar{g} \\
e & 0 & \bar{h} & \bar{l} \\
f & h & 0 & \bar{m} \\
g & l & m & 0
\end{array}\right) \text { and } \alpha=\frac{2 r}{t}=\frac{2 \sqrt{5}}{\sqrt{3}} .
$$

Using the same notations as before, equations (2) and (5) can be written as follows

$$
A \mathbf{1}=-\vec{X}
$$

Substituting (6.11) into (6.10) one obtains that

$$
\left(A^{2}-\alpha A-I\right) \mathbf{1}=\mathbf{0} .
$$

Matrix $A$ is Hermitian and so it is diagonalizable and has a complete orthonormal set of eigenvectors. Suppose $\lambda$ is an eigenvalue for $A$ corresponding to the eigenvector $\vec{\psi}$. Then we have that

$$
A \vec{\psi}=\lambda \vec{\psi}, \quad \Leftrightarrow \quad\left(A^{2}-\alpha A-I\right) \vec{\psi}=\left(\lambda^{2}-\alpha \lambda-1\right) \vec{\psi} .
$$


From equations (6.12) and (6.13) it follows that 1 belongs to the kernel of $A^{2}-$ $\alpha A-I$ if and only if it is the eigenvector for $A$ corresponding to the eigenvalue $\lambda$ satisfying $\lambda^{2}-\alpha \lambda-1=0$. Therefore

$$
A \mathbf{1}=\lambda_{ \pm} \mathbf{1}
$$

where $\lambda_{ \pm}=\frac{\sqrt{5} \pm 2 \sqrt{2}}{\sqrt{3}}$. Comparing equations (6.11) and (6.14) one obtains that

$$
\vec{X}=-\lambda_{ \pm} \mathbf{1}
$$

Substituting the values of the parameters $a, b, c, d$ obtained here into equation (1) of the system (6.9) one obtains the contradiction that

$$
\pm 4 \lambda_{ \pm}=0
$$

This result shows that if $\left(n^{+}, v^{+}\right)=(2,1)$, then no nontrivial ET-matrix exists.

5. Case $(4,5)$. This case yields the same values of $r$ and $t$ as case $(2,1)$ above. The corresponding matrices is given by

$$
C=\left(\begin{array}{cccccc}
r & t & t & t & t & t \\
t & r & a t & b t & c t & d t \\
t & \bar{a} t & r & e t & f t & g t \\
t & \bar{b} t & \bar{e} t & r & h t & l t \\
t & \bar{c} t & \bar{f} t & \bar{h} t & r & m t \\
t & \bar{d} t & \bar{g} t & \bar{l} t & \bar{m} t & -r
\end{array}\right)
$$

where

$$
a, b, c, d, e, f, g, h, l, m \in\{z \in \mathbb{C}:|z|=1\} .
$$

From the orthogonality of the rows of $C$ we obtain the following system of equations

$$
\begin{gathered}
\left\{\begin{array} { l l } 
{ 2 r + t ( a + b + c + d ) = 0 } & { ( 1 ) } \\
{ 2 r + t ( \overline { a } + e + f + g ) = 0 } & { ( 2 ) } \\
{ 2 r + t ( \overline { b } + \overline { e } + h + l ) = 0 } & { ( 3 ) } \\
{ 2 r + t ( \overline { c } + \overline { f } + \overline { h } + m ) = 0 } & { ( 4 ) } \\
{ \overline { d } + \overline { g } + \overline { l } + \overline { m } = 0 } & { ( 5 ) }
\end{array} \quad \left\{\begin{array}{l}
2 \bar{a} r+t(1+\bar{b} e+\bar{c} f+\bar{d} g)=0 \\
2 \bar{a} r+t(1+\overline{a e}+\bar{c} h+\bar{d} l)=0 \\
2 \bar{b} r+t(1+\bar{a} \bar{f}+\overline{b h}+\bar{d} m)=0 \\
1+\overline{a g}+\overline{b l}+\overline{c m}=0
\end{array}\right.\right. \\
\left\{\begin{array} { l l } 
{ 2 \overline { e } r + t ( 1 + a \overline { b } + \overline { f } h + \overline { g } l ) = 0 } & { ( 1 0 ) } \\
{ 2 \overline { f } r + t ( 1 + a \overline { c } + \overline { e } \overline { h } + \overline { g } m ) = 0 } & { ( 1 1 ) } \\
{ 1 + a \overline { d } + \overline { e } \overline { l } + \overline { f } \overline { m } = 0 } & { ( 1 2 ) }
\end{array} \quad \left\{\begin{array}{l}
2 \bar{h} r+t(1+b \bar{c}+e \bar{f}+\bar{l} m)=0 \\
1+b \bar{d}+e \bar{g}+\bar{h} \bar{m}=0 \\
1+c \bar{d}+f \bar{g}+h \bar{l}=0
\end{array}\right.\right.
\end{gathered}
$$

This case is similar to case $(2,1)$ and can be analysed in a similar fashion with the following modifications

1. From equations (1) to (4) matrix $A$ and vector $\vec{X}$ are obtained respectively as

$$
\left(\begin{array}{cccc}
0 & a & b & c \\
\bar{a} & 0 & e & f \\
\bar{b} & \bar{e} & 0 & h \\
\bar{c} & \bar{f} & \bar{h} & 0
\end{array}\right) \quad \text { and } \quad\left(\begin{array}{c}
d \\
g \\
l \\
m
\end{array}\right)
$$

2. Equations (9), (12), (14) and (15) yield the matrix equation $A \vec{X}=-\mathbf{1}$. 
The analysis shows that

$$
\vec{X}=-\frac{1}{\lambda_{ \pm}} \mathbf{1}, \quad \lambda_{ \pm}=\frac{\sqrt{5} \pm \sqrt{2}}{\sqrt{3}} .
$$

Substituting the corresponding values of the parameters into equation (5) of the system (6.16) yields the contradiction that $-4 \frac{1}{\lambda_{ \pm}}=0$. Thus no nontrivial ET-matix exists in this case as well.

6. Case $(3,3)$. Here the trace of $C$ is zero and the value of $r$ cannot be determined from the formula (3.2). Since the number of positive and negative diagonal entries are equal, the corresponding matrix $C$ can be represented as

$$
C=\left(\begin{array}{cccccc}
r & t & t & t & t & t \\
t & r & a t & b t & c t & d t \\
t & \bar{a} t & r & e t & f t & g t \\
t & \bar{b} t & \bar{e} t & -r & h t & l t \\
t & \bar{c} t & \bar{f} t & \bar{h} t & -r & m t \\
t & \bar{d} t & \bar{g} t & \bar{l} t & \bar{m} t & -r
\end{array}\right)
$$

where

$$
a, b, c, d, e, f, g, h, l, m \in\{z \in \mathbb{C}:|z|=1\} .
$$

From the orthogonality of the rows of $C$ we obtain the following system of equations

$$
\begin{array}{cl} 
\begin{cases}2 r+t(a+b+c+d)=0 & (1) \\
2 r+t(\bar{a}+e+f+g)=0 & (2) \\
\bar{b}+\bar{e}+h+l=0 & (3) \\
\bar{c}+\bar{f}+\bar{h}+m=0 & (4) \\
\bar{d}+\bar{g}+\bar{l}+\bar{m}=0 & (5)\end{cases} & \left\{\begin{array}{l}
2 \bar{a} r+t(1+\bar{b} e+\bar{c} f+\bar{d} g)=0 \\
1+\overline{a e}+\bar{c} h+\bar{d} l=0 \\
1+\bar{a} \bar{f}+\overline{b h}+\bar{d} m=0 \\
1+\overline{a g}+\overline{b l}+\overline{c m}=0
\end{array}\right. \\
\begin{cases}1+a \bar{b}+\bar{f} h+\bar{g} l=0 & (10) \\
1+a \bar{c}+\bar{e} \bar{h}+\bar{g} m=0 & (11) \\
1+a \bar{d}+\bar{e} \bar{l}+\bar{f} \bar{m}=0 & (12)\end{cases} & \left\{\begin{array}{l}
2 \bar{h} r+t(1+b \bar{c}+e \bar{f}+\bar{l} m)=0 \\
-2 \bar{l} r+t(1+b \bar{d}+e \bar{g}+\bar{h} \bar{m})=0
\end{array}\right. \\
& \{2 \bar{m} r+t(1+c \bar{d}+f \bar{g}+h \bar{l})=0
\end{array}
$$

In this system there are nine equations to which Lemma 1 can be applied directly: equations (3), (4), (5), (7), (8), (9), (10), (11) and (12). It should be noted that the application of the Lemma is done to an appropriate equation in each subsequent system, starting with any of the nine equations in the above system. For example applying the Lemma to equation (3) yields three possibilities. Making substitutions from any of the possibilities yields an equivalent system in which equation (3) vanishes. In that subsequent system, the number of equations to which the Lemma can now be applied will be at most eight.

The details of the computations in determining the parameters in this case can be found in the appendix B. The results can be grouped into four categories depending the kind of matrix $C$ obtained:

(a) no matrix exists,

(b) unique real matrix,

(c) one parameter family,

(d) two parameters family. 
Below we give examples from the last three categories.

Example 1 (Unique real matrix). This result is obtained by making the following choices when Lemma 1 is applied to equations (3), (4) and (5) respectively of the system (6.17)

$$
\left\{\begin{array}{l}
h=-\bar{b} \\
l=-\bar{e}
\end{array}, \quad\left\{\begin{array} { l } 
{ c = - h } \\
{ m = - \overline { f } }
\end{array} \quad \text { and } \quad \left\{\begin{array}{l}
l=-d \\
m=-g .
\end{array}\right.\right.\right.
$$

With these substitutions, the equations (3), (4), and (5) vanish from the system and it can also be seen that $c=\bar{b}, e=\bar{d}$ and $g=\bar{f}$. Equation (7) can now be written as $\bar{a} d-1=0$ which implies that $d=a$. At this stage, other parameters that can be expressed in terms of $a$ are $e=\bar{a}$ and $l=-a$. Equations (1) and (2) of the system (6.17) now become

$$
2 r+t(2 a+b+\bar{b})=0 \quad(1) \quad 2 r+t(2 \bar{a}+f+\bar{f})=0
$$

Evaluating the difference of equation (1) and the conjugate of equation (2) yields that $\mathfrak{R}(f)=\mathfrak{R}(b)$ which means that either $f=b$ or $f=\bar{b}$. Choosing $f=b$ and the other relations above, equations (6) and (13) of the system (6.17) now become

$$
2 \bar{a} r+t\left(1+2 \bar{a} \bar{b}+b^{2}\right)=0 \quad(6) \quad 2 b r+t\left(1+b^{2}+2 \bar{a} \bar{b}\right)=0
$$

The difference of these two equations shows that $b=\bar{a}$. With this value of $b$, equation (1) is now written as $r+t(a+\Re(b))=0$. This relation between $r$ and $t$ and that in equation (3.1) show that

$$
t=\frac{1}{\sqrt{5+(a+\Re(a))^{2}}} \quad \text { and } \quad r=\frac{-(a+\Re(a))}{\sqrt{5+(a+\Re(a))^{2}}} .
$$

Since $r$ is real, $a$ is necessarily real implying that $a= \pm 1$. The appropriate choice of $a$ so that $r$ is nonnegative is $a=-1$. Thus the matrix $C$ is fully determined and real as seen below

$$
C=\frac{1}{3}\left(\begin{array}{cccccc}
2 & 1 & 1 & 1 & 1 & 1 \\
1 & 2 & -1 & -1 & -1 & -1 \\
1 & -1 & 2 & -1 & -1 & -1 \\
1 & -1 & -1 & -2 & 1 & 1 \\
1 & -1 & -1 & 1 & -2 & 1 \\
1 & -1 & -1 & 1 & 1 & -2
\end{array}\right)
$$

Example 2 (One parameter family). Suppose in Example 1 above the relation $f=\bar{b}$ is chosen instead of $f=b$, while the other relations are retained. Then $a$ and $b$ are arbitrary and equations (1), (13) and (15) of the system (6.17) can be written as follows

$$
\begin{array}{r}
2 r+t(2 a+b+\bar{b})=0 \\
2 b r+t\left(1+b^{2}+2 \bar{a} b\right)=0 \\
2 \bar{b} r+t\left(1+2 \bar{a} \bar{b}+\bar{b}^{2}\right)=0
\end{array}
$$

Equation (1) then takes the form $r+t(a+\Re(b))=0$. Since $r$ and $t$ are real, $a$ is also real meaning that $a= \pm 1$. The resulting relation between $r$ and $t$ and equation (3.1) yield that

$$
t=\frac{1}{\sqrt{5+\alpha^{2}}} \quad \text { and } \quad r=\frac{-\alpha}{\sqrt{5+\alpha^{2}}}
$$

where $\alpha= \pm 1+\Re(b)$. 
Since $r>0, \alpha$ should be chosen negative and this is obtained when $a=-1$. The sign of $\Re(b)$ does not determine the sign of $\alpha$ because $|\Re(b)| \leq 1$. In particular the value $b=1$ is not allowed because then one obtains that $r=0$ which is excluded. Thus $\alpha=-1+\Re(b)$. The ET-matrix $C$ is fully determined and is given in terms of $b$ as seen below

$$
C=\frac{1}{\sqrt{5+(1-\Re(b))^{2}}}\left(\begin{array}{cccccc}
1-\Re(b) & 1 & 1 & 1 & 1 & 1 \\
1 & 1-\Re(b) & -1 & b & \bar{b} & -1 \\
1 & -1 & 1-\Re(b) & -1 & \bar{b} & b \\
1 & \bar{b} & -1 & -1+\Re(b) & -\bar{b} & 1 \\
1 & b & b & -b & -1+\Re(b) & -b \\
1 & -1 & \bar{b} & 1 & -\bar{b} & -1+\Re(b)
\end{array}\right) .
$$

Example 3 (Two parameters family). Here the choices made when Lemma 1 is applied to equation (3), (4) and (5) respectively of the system (6.17) are

$$
\left\{\begin{array}{l}
h=-\bar{b} \\
l=-\bar{e}
\end{array} \quad, \quad\left\{\begin{array} { l } 
{ f = - h } \\
{ m = - \overline { c } }
\end{array} \quad \text { and } \left\{\begin{array}{l}
g=-m \\
l=-d
\end{array}\right.\right. \text {. }\right.
$$

The following relations immediately emerge; $e=\bar{d}, f=\bar{b}$ and $g=\bar{c}$. With these relations the system (6.16) reduces to the following

$$
\left\{\begin{array}{l}
2 r+t(a+b+c+d)=0 \\
\bar{a} d-\bar{b} \bar{c}=0
\end{array}\right.
$$

From the first equation in (6.20) and from equation (3.1), the values of $r$ and $t$ in terms of $a, b, c, d$ are

$$
r=\frac{-\alpha}{\sqrt{20+\alpha^{2}}}, \quad t=\frac{2}{\sqrt{20+\alpha^{2}}},
$$

where $\alpha=a+b+c+d$. Since $r$ and $t$ are real and nonnegative, $\alpha$ has to be chosen real and negative. Remembering that the parameters are unimodular, the system (6.20) can now be written as

$$
\left\{\begin{array}{l}
a+b+c+d=\alpha \in \mathbb{R}_{-} \\
a=b c d
\end{array}\right.
$$

where $\mathbb{R}_{-}=(-\infty, 0)$. These equations can be written as a linear system of $a$ and $d$ as follows

$$
\left\{\begin{array}{l}
a+d=\alpha-b-c \\
a-b c d=0 .
\end{array}\right.
$$

The system yields values of $a$ and $d$ if and only if the determinant of the coefficients matrix does not vanish. Thus we require that $b c \neq-1$. The values of $a$ and $d$ are then obtained as

$$
a=\frac{b c(\alpha-b-c)}{1+b c}, \quad d=\frac{\alpha-b-c}{1+b c} .
$$

Given that $d$ is unimodular, it follows that $d \bar{d}=1$. Substituting the value of $d$ above into this equation and simplifying one obtains that

$$
\alpha^{2}-2 \alpha \Re(b+c)+4 \mathfrak{I}(b) \mathfrak{I}(c)=0
$$

from which it follows that

$$
\alpha=\Re(b+c) \pm \sqrt{(\Re(b+c))^{2}-4 \mathfrak{I}(b) \mathfrak{I}(c) .}
$$


The value $\alpha \in \mathbb{R}_{-}$is obtained in any of the following cases

1. $(\mathfrak{R}(b+c))^{2}-4 \mathfrak{I}(b) \mathfrak{I}(c)>0$ and $\mathfrak{I}(b) \mathfrak{I}(c)<0$. Here there exists a unique $\alpha \in \mathbb{R}_{-}$.

2. $(\mathfrak{R}(b+c))^{2}-4 \mathfrak{I}(b) \mathfrak{I}(c)>0, \mathfrak{I}(b) \mathfrak{I}(c)>0$ and $\mathfrak{R}(b+c)<0$. There exists two $\alpha \in \mathbb{R}_{-}$.

3. $(\mathfrak{R}(b+c))^{2}-4 \mathfrak{I}(b) \mathfrak{I}(c)=0, \mathfrak{I}(b) \mathfrak{I}(c)>0$ and $\mathfrak{R}(b+c)<0$. There exists a unique $\alpha \in \mathbb{R}_{-}$.

4. $\mathfrak{I}(b) \mathfrak{I}(c)=0$ and $\mathfrak{R}(b+c)<0$. There exists a unique $\alpha \in \mathbb{R}_{-}$since $\alpha=0$ is excluded.

The corresponding matrix $C$ can now be written as follows

$$
C=\frac{1}{\sqrt{20+\alpha^{2}}}\left(\begin{array}{cccccc}
-\alpha & 2 & 2 & 2 & 2 & 2 \\
2 & -\alpha & 2 a & 2 b & 2 c & 2 d \\
2 & 2 \bar{a} & -\alpha & -2 c & 2 \bar{b} & -2 d \\
2 & 2 \bar{b} & -2 \bar{c} & \alpha & -2 \bar{b} & 2 \bar{c} \\
2 & 2 \bar{c} & 2 b & -2 b & \alpha & -2 \bar{c} \\
2 & 2 \bar{d} & -2 \bar{d} & 2 c & -2 c & \alpha
\end{array}\right)
$$

Here $b$ and $c$ are arbitrary parameters subject to $b c \neq-1, \alpha$ is given by the formula (6.24) and $a$ and $d$ by the equations (6.23).

On the other hand, suppose that $b c=-1$ is allowed. Then $c=-\bar{b}$ and the system (6.20) now becomes

$$
\left\{\begin{array}{l}
a+d=\alpha-b+\bar{b} \\
a+d=0
\end{array}\right.
$$

This implies that $\alpha=b-\bar{b}$. Since $\alpha \in \mathbb{R}$ it follows that $\alpha=0$ contrary to the requirement that $\alpha$ is negative.

Conclusion. If $\operatorname{Tr}(S) \neq 0$, there are few possible values of $r$ given by the formula (3.2). It appears that almost all such $r$ do not yield an ET-matrix. Our calculations show that only the same $r$ as for the standard matching conditions is possible since such matrices are equivalent to standard matching conditions scattering matrices.

If $\operatorname{Tr}(S)=0$, then $r$ cannot be determined by equation (3.2). This case occurs only for matrices of even dimension and the corresponding ET-matrices exist as follows. When $n=2,4$, there exixt one parameter families of matrices $C$. When $n=6$, there exists a unique real matrix $C$ which is equivalent to the SMC-matrix, a one-parameter family and two-parameter family matrices $C$.

We have described all possible ET-matrices in dimensions 2 to 6 . Before only examples of such matrices were constructed. Our hypothesis is that in all dimensions for matrices having representation (2.1) with zero trace, and this occurs when the oder of the matrix is even, there exist some families of ET-matrices. In odd dimensions there exists only those generated by the SMC-matrix

\section{References}

[1] G. Berkolaiko and P. Kuchment Introduction to quantum graphs, American Mathematical Society, Rhode Island, 2013.

[2] M. Hirobumi and S. Iwao The scattering matrix, Electronic Journal of Combinatorics, 15 (2008).

[3] G. Berkolaiko, J. M. Harrison and J. H. Wilson Mathematical aspects of vacuum energy on quantum graphs, Journal of Physics. A. Mathematical and Theoritical, 42 (2009).

[4] R. Band, J. M. Harrison and C. H. Joyner Finite pseudo orbit expansions for spectral quantities of quantum graphs, Journal of Physics. A. Mathematical and Theoritical, 45 (2012).

[5] T. Ondřej and C. Taksu Quantum graph vertices with permutation-symmetric scattering probabilities, Physics Letters. A, 375 (2011).

[6] T. Ondřej, P. Exner and C. Taksu Reflectioless and equiscattering quantum graphs, IARIA, (2001). 
[7] T. Ondřej and C. Taksu Hermitian unitary matrices with modular permutation symmetry, arXiv, (2011)

[8] T. Cheon, Reflectionless and Equiscattering Quantum Graphs and Their Applications, Int. J. System \& Measurement, 5 (2012) 34-44.

[9] T. Cheon, Reflectionless and Equiscattering Quantum Graphs, textitInt. J. Adv. Systems and Measurements, 5 (2012), 18-22.

[10] M. Harmer, Hermitian symplectic geometry and extension theory, Journal of Physics. A. Mathematical and General, 33, (2000),9193-9203.

[11] M. Harmer, Hermitian symplectic geometry and the factorization of the scattering matrix on graphs, Journal of Physics. A. Mathematical and General, 33, (2000),9015-9032.

[12] J.M. Harrison, U. Smilansky, and B. Winn, Quantum graphs where back-scattering is prohibited, Journal of Physics. A. Mathematical and Theoritical, 40, (2007), 14181-14193.

[13] R.B. Holmes and V.I. Paulsen, Optimal frames for erasures, Linear Algebra Appl. 377 (2004), 31 ï¡ 51.

[14] P. Kurasov, Quantum graphs:Spectral theory and inverse problems, 2014 monograph in preparation.

[15] P. Kurasov and M. Nowaczyk, Geometric properties of quantum graphs and vertex scattering matrices, Opuscula Mathematica, 30, (2010).

[16] P. Kurasov, R. Ogik and A. Rauf, On reflectionless equi-transmitting matrices, Institut Mittag-Leffler Report No. 24 2012/2013, https://www.mittag-leffler.se/preprints/files/IML-1213s-24.pdf

[17] O. Post, Spectral analysis on graph-like spaces, Springer, 2010.

[18] O. Turek and T. Cheon, Quantum graph vertices with permutation-symmetric scattering probabilities, Phys. Lett. A 375 (2011), no. 43, 3775ï $; 3780$.

[19] O. Turek and T. Cheon, Hermitian unitary matrices with modular permutation symmetry, arXiv:1104.0408.

Dept. of Mathematics, Stockholm Univ. 10691 Stockholm, SWEDEN 